\title{
Construction of Quantum LDPC Codes From Classical Row-Circulant QC-LDPCs
}

\author{
Zunaira Babar, Panagiotis Botsinis, Soon Xin Ng, Dimitrios Alanis, and Lajos Hanzo
}

Abstract-Classical row-circulant quasi-cyclic (QC) low-density parity check (LDPC) matrices are known to generate efficient high-rate short and moderate-length QC-LDPC codes, while the comparable random structures exhibit numerous short cycles of length-4. Therefore, we conceive a general formalism for constructing nondual-containing Calderbank-Shor-Steane (CSS)type quantum low-density parity check (QLDPC) codes from arbitrary classical row-circulant QC-LDPC matrices. We apply our proposed formalism to the classical balanced incomplete block design (BIBD)-based row-circulant QC-LDPC codes for demonstrating that our designed codes outperform their dual-containing CSS-type counterparts as well as the entanglement-assisted (EA)QLDPC codes.
\end{abstract}

Index Terms-Quantum error correction, low density parity check codes, iterative decoding.

$\mathbf{Q}$ UANTUM-domain parallel processing provides a plausible solution for achieving full-search based multi-stream detection [1], which is vital for future gigabitwireless systems. The peculiar laws of quantum mechanics have also spurred interest in the absolutely-secure quantumbased communication systems [2], [3]. Unfortunately, quantum decoherence imposes a hitherto insurmountable impairment on the practical implementation of quantum computation as well as on quantum communication systems. More specifically, decoherence perturbs the fragile quantum states, which may be characterized either by bit-flips or phase-flips - or in fact possibly both - inflicted on the constituent qubits. Analogously to the classical error correction codes, these detrimental effects of decoherence may be overcome with the aid of efficient quantum error correction codes (QECCs).

Meritorious families of QECCs can be constructed from the known classical binary as well as quaternary codes by invoking the stabilizer formalism [4]. In particular, the astounding performance of the classical low density parity check (LDPC) codes achieved at an affordable decoding complexity has inspired the community to construct their stabilizer-based quantum counterparts, i.e. quantum low density parity check (QLDPC) codes. In

\footnotetext{
Manuscript received June 22, 2015; accepted October 20, 2015. The associate editor coordinating the review of this paper and approving it for publication was H. Saeedi.

The authors are with the University of Southhampton, Southampton SO16 1BJ, U.K. (e-mail: zb2g10@ecs.soton.ac.uk).

Digital Object Identifier 10.1109/LCOMM.2015.2494020
}

this letter, we focus our attention on the construction of QLDPC codes from the family of classical row-circulant quasi-cyclic (QC)-LDPC matrices, which are known to generate efficient high-rate short and moderate-length QC-LDPC codes [5]-[7]. The balanced incomplete block design (BIBD) [6] and the cyclic difference family based LDPC code structures [7] are particularly significant in this respect. The resultant classical codes have at least a girth of 6 , while the randomly constructed LDPC codes of comparable code length have numerous cycles of length 4, which impair the performance of the associated decoding algorithm.

In [8], Mackay et al. presented generalized methods, namely 'bicycle' and 'unicycle' codes, for constructing dual-containing Calderbank-Shor-Steane (CSS)-type QLDPC codes from arbitrary classical LDPCs. Later Djordjevic [9] extended these ideas for constructing the family of BIBD-based high-rate QLDPC codes. Unfortunately, these dual-containing CSS structures have numerous unavoidable short cycles of length4. Inspired by the concept of entanglement-assisted (EA)QLDPC codes [10], which do not exhibit the unavoidable length-4 cycles in the binary formalism, Djordjevic [11] conceived the EA counterparts of the BIBD-based designs of [9]. The resultant EA-QLDPC codes required a single preshared entangled qubit (ebit), which constitutes a valuable resource, because maintaining a noiseless entangled state is not a trivial task. Furthermore, both the dual-containing CSS codes as well as the EA-QLDPC codes of [9] constitute a class of homogeneous CSS codes, which have numerous short cycles in the Galois field GF(4) formalism. In contrast to these developments, we propose a general formalism for constructing non-dual-containing CSS-type QLDPC codes from arbitrary classical row-circulant $Q C-L D P C$ matrices. The proposed construction brings with it the following plausible benefits:

- Since the constructed codes are non-dual-containing, they do not suffer from having unavoidable short cycles in the binary formalism and have fewer short cycles in the GF(4) formalism than their homogeneous counterparts.

- Pre-shared ebits are not required.

We apply our proposed methodology to the family of classical $B I B D$-based row-circulant $Q C$-LDPC codes for evaluating the performance of the resultant QLDPC codes.

This letter is organized as follows. In Section II, we review the BIBD-based classical Row-Circulant QC-LDPC codes, while the proposed construction method is presented in Section III. Our results are discussed in Section IV, while our conclusions are offered in Section V. 


\section{Classical BIBD-Based Row-Circulant QC-LDPCS}

The BIBD constructions proposed by Bose [12] constitute the family of classical row-circulant QC-LDPC codes. A BIBD characterized by the parameters $(v, b, r, k, \lambda)$ divides all the $v$ elements of a set $V$ into $b$ blocks of size $k$ so that each pair of elements occurs in exactly $\lambda$ of the blocks, whilst every element occurs in exactly $r$ blocks and the number of elements $k$ in each block is small as compared to the size $v$ of the set $V$. Based on this notation, Bose proposed the following BIBD [12] constructions, which are suitable for conceiving the row-circulant QC-LDPCs [6].

1) Type-I Bose BIBDs: Given that $t$ is a positive integer so that $(12 t+1)$ is a power of a prime, then there exists a prime Galois field $\operatorname{GF}(12 t+1)$ having elements ranging from 0 to $12 t$, which constitute the finite set $V$ of the BIBD. Furthermore, let $\alpha$ be the primitive element of $\operatorname{GF}(12 t+1)$, which satisfies the following condition:

$$
\alpha^{4 \mathrm{t}}-1=\alpha^{\mathrm{c}}
$$

where $c$ is an integer in the range $0<c<12 t+1$. Based on this notation, Bose [12] proposed that there exists a BIBD having the parameters $v=(12 \mathrm{t}+1), b=\mathrm{t}(12 \mathrm{t}+1), r=4 \mathrm{t}$, $k=4$ and $\lambda=1$, whose $\mathrm{t}$ base blocks are given by:

$$
B_{i}=\left\{0, \alpha^{2 i}, \alpha^{2 i+4 \mathrm{t}}, \alpha^{2 i+8 \mathrm{t}}\right\},
$$

for $0 \leq i<\mathrm{t}$. We can then proceed by creating $(12 \mathrm{t}+1)$ blocks from the base block $B_{i}$ by adding each element of the Galois field to each element of the base block, hence creating a total of $t(12 t+1)$ blocks. The incidence matrix of this BIBD is a $(12 t+1) \times t(12 t+1)$ matrix, which is as follows:

$$
H_{\mathrm{BIBD}}=\left(H_{0}, H_{1}, \ldots, H_{\mathrm{t}-1}\right),
$$

where the $i$ th submatrix $H_{i}$ is a $(12 t+1) \times(12 t+1)-$ element circulant matrix corresponding to the base block $B_{i}$. Furthermore, the matrix $H_{\text {BIBD }}$ has a row weight of $4 \mathrm{t}$ and a column weight of 4. Since the incidence matrix of Eq. (3) has the required properties of a QC-LDPC matrix, a subarray of $H_{\mathrm{BIBD}}$, having $m$ submatrices for $0<m<t$, can be used for constructing a classical row-circulant $(12 t+1) \times m(12 t+1)$-element QC-LDPC matrix. The minimum distance of the resultant LDPC code is at least 5 and the coding rate is approximately $(m-1) / m$.

2) Type-II Bose BIBDs: Let $t$ be a positive integer so that $(20 t+1)$ is a power of a prime, then there exists a prime Galois field $\mathrm{GF}(20 t+1)$ having elements ranging from 0 to $20 t$, which constitute the finite set $V$ of the BIBD. In contrast to Eq. (1), the primitive element $\alpha$ of $\mathrm{GF}(20 t+1)$ has to satisfy the condition:

$$
\alpha^{4 \mathrm{t}}+1=\alpha^{\mathrm{c}}
$$

where $c$ is an integer in the range $0<c<20 t+1$. Bose [12] proposed that we can construct a BIBD having the parameters of $v=(20 \mathrm{t}+1), b=\mathrm{t}(20 \mathrm{t}+1), r=5 \mathrm{t}, k=5$ and $\lambda=1$, whose $t$ base blocks are given by:

$$
B_{i}=\left\{\alpha^{2 i}, \alpha^{2 i+4 \mathrm{t}}, \alpha^{2 i+8 \mathrm{t}}, \alpha^{2 i+12 \mathrm{t}}, \alpha^{2 i+16 \mathrm{t}}\right\},
$$

for $0 \leq i<\mathrm{t}$. Similar to the Type-I design, $(12 \mathrm{t}+1)$ blocks 136 can be constructed for each base block $B_{i}$. The incidence matrix 137 of the resultant BIBD is a $(20 t+1) \times t(20 t+1)$-element 138 matrix, which is formed by t submatrices, as previously shown 139 in Eq. (3). For the Type-II design, the matrix $H_{\text {BIBD }}$ has a row 140 weight of 5t and a column weight of 5. Again, we can construct 141 a row-circulant QC-LDPC of size $(20 t+1) \times m(20 t+1)$ by 142 using a subarray of $H_{\mathrm{BIBD}}$.

\section{Proposed QLDPC Code Construction}

The family of CSS codes, invented independently by 145 Calderbank and Shor [13] as well as by Steane [14], con- 146 stitute a special class of stabilizer codes, which facilitate the 147 design of quantum codes from a pair of classical binary codes. More explicitly, an $\left[n, k_{1}-k_{2}\right]$ CSS code, which is capable of correcting $(d-1) / 2$ bit-flips as well as phase-flips, can be constructed from the classical linear block codes $C_{1}\left(n, k_{1}\right)$ and $C_{2}\left(n, k_{2}\right)$, provided that we have $C_{2} \subset C_{1}$, and that $C_{1}$ as well as the dual of $C_{2}$, i.e. $C_{2}^{\perp}$, have a minimum Hamming distance of $d$. If $H_{z}$ and $H_{x}$ are the parity check matrices (PCMs) of $C_{1}$ and $C_{2}^{\perp}$, respectively, then the resultant CSS code assumes the following form [8]:

$$
\left(\begin{array}{cc}
H_{z} & 0 \\
0 & H_{x}
\end{array}\right) .
$$

More explicitly, $H_{z}$ is used for bit-flip correction, while $H_{x}$ cor- 157 rects the phase-flips. Furthermore, since $C_{2} \subset C_{1}$, the PCMs 158 $H_{z}$ and $H_{x}$ must satisfy the symplectic criterion, which may be 159 defined as:

$$
H_{z} H_{x}^{T}=0
$$

When $H_{z}=H_{x}$, the constraint of Eq. (7) reduces to $H_{z} H_{z}^{T}=161$ 0 , which is referred to as a 'dual-containing' CSS code. In 162 the context of the QLDPC codes, this dual-containing structure 163 results in numerous unavoidable short cycles in the associated 164 Tanner graph, which in turn degrade the performance of the 165 decoding algorithm. Furthermore, if the symplectic criterion is 166 not intrinsically satisfied, then pre-shared ebits may be used. 167 The resultant codes constitute the family of EA codes.

We may also view the PCM of Eq. (6) in the GF(4) formalism 169 as [15]:

$$
\hat{H}=\left(\begin{array}{c}
\omega H_{z} \\
H_{x}
\end{array}\right),
$$

where $\{0,1, \omega, \bar{\omega}\} \in \mathrm{GF}(4)$. When both $H_{z}$ and $H_{x}$ are the 171 same, as in the family of dual-containing CSS and EA-QLDPC 172 codes $^{1}$, we may refer to them as homogeneous CSS codes. The 173 $(\mathrm{m} \times n)$-element ${ }^{2}$ PCM $\hat{H}$ of a homogeneous code exhibits 174 numerous short cycles in its Tanner graph, because the $i$ th and 175 $(i+\mathrm{m} / 2)$ th rows completely overlap, i.e. they have non-zero 176 values at the same indices. These short cycles, resulting from 177

\footnotetext{
${ }^{1}$ To the best of our knowledge, all the CSS-type EA-QLDPC codes proposed to date are homogeneous.

${ }^{2}$ Please note that $\mathrm{m}$ denotes the number of rows of the PCM $\hat{H}$, while $m$ represents the number of submatrices of $H_{\mathrm{BIBD}}$ used in an LDPC matrix.
} 
the global homogeneous code structure, appear in addition to the short cycles within the local structures of the PCMs $H_{z}$ and $H_{x}$, or, equivalently, in addition to the short cycles observed in the binary formalism of Eq. (6). It is pertinent to mention here that short cycles in the GF(4) formalism are a by-product of the symplectic criterion of Eq. (7), which cannot be completely eliminated even if both $H_{z}$ and $H_{x}$ have a girth of 6 in the binary formalism [16]. However, the number of short cycles may be reduced by adopting a non-dual-containing (or equivalently non-homogeneous) design, having $H_{x} \neq H_{z}$.

In contrast to both the dual-containing and to the EA structures, which suffer from numerous unavoidable short cycles, non-dual-containing codes may be designed, so that they have at least a girth of 6 in the binary formalism of Eq. (6) and fewer short cycles in the GF(4) formalism of Eq. (8) by virtue of their non-homogeneous nature. Therefore, we focus our attention on the non-dual-containing structure for constructing QLDPC codes from the pair of classical row-circulant QCLDPC matrices $H_{z}$ and $H_{x}$, each having a girth of at least 6 . Let us consider a row-circulant QC-LDPC matrix $H$, which is a subarray of $H_{\text {BIBD }}$ of Eq. (3), assuming that it consists of an even number of square circulant submatrices. Inspired by the non-dual-containing CSS-type QC-QLDPC codes of [17], we propose that if we formulate $H_{z}$ and $H_{x}$ as follows:

$$
\begin{aligned}
& H_{z}=H \\
& H_{x}=\left(H_{\frac{m}{2}}^{T}, H_{\frac{m}{2}+1}^{T}, \ldots, H_{m-1}^{T}, H_{0}^{T}, H_{1}^{T}, \ldots, H_{\frac{m}{2}-1}^{T}\right),
\end{aligned}
$$

where $m$ is even, then the resultant CSS code satisfies the symplectic criterion of $H_{z} H_{x}^{T}=0$. This may be readily shown as follows:

$$
\begin{aligned}
& \left(H_{0}, H_{1}, \ldots, H_{\frac{m}{2}-1}, H_{\frac{m}{2}}, H_{\frac{m}{2}+1}, \ldots, H_{m-1}\right)\left(\begin{array}{c}
H_{\frac{m}{2}} \\
H_{\frac{m}{2}+1} \\
\vdots \\
H_{m-1} \\
H_{0} \\
H_{1} \\
\vdots \\
H_{\frac{m}{2}-1}
\end{array}\right) \\
& =H_{0} H_{\frac{m}{2}}+H_{1} H_{\frac{m}{2}+1}+\cdots+H_{\frac{m}{2}-1} H_{m-1} \\
& +H_{\frac{m}{2}} H_{0}+H_{\frac{m}{2}+1} H_{1}+\cdots+H_{m-1} H_{\frac{m}{2}-1} \text {. }
\end{aligned}
$$

Since the multiplication of circulant matrices is commutative, the two parts of Eq. (10), i.e. $\left(H_{0} H_{\frac{m}{2}}+H_{1} H_{\frac{m}{2}}+1+\right.$ $\left.\cdots+H_{\frac{m}{2}} H_{m}\right)$ and $\left(H_{\frac{m}{2}} H_{0}+H_{\frac{m}{2}+1} H_{1}+\cdots+H_{m-1} H_{\frac{m}{2}-1}\right)$ are equal. Hence, the modulo $2^{2}$ addition of Eq. (10) yields 0 ; thus, satisfying the symplectic criterion. Furthermore, the resultant quantum coding rate is approximately $(m-2) / m$.

Let us now scrutinize the girth of the PCMs $H_{z}$ and $H_{x}$ in the binary formalism. The constituent $(l \times l)$-element circulant submatrix $H_{i}$ of Eq. (3), which has a row weight and a column weight of $\gamma$, is uniquely and unambiguously characterized by the polynomial $h_{i}(x)=x^{d_{i, 0}}+x^{d_{i, 1}}+\cdots+x^{d_{i, \gamma-1}}$, where $d_{i, j}$ denotes the column index of the $j$ th non-zero entry in the first row of $H_{i}$. For example, if the first row of $H_{i}$

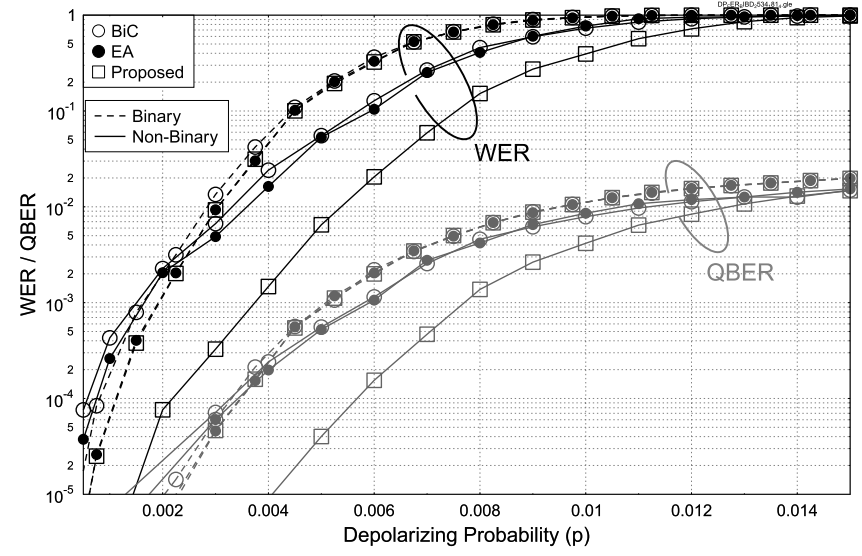

Fig. 1. Comparison of the achievable WER/QBER performance of our proposed [2534, 2172] QLDPC code (labeled 'Proposed') with the bicycle code of Eq. (11) (labeled 'BiC') and the EA-QLDPC code of Eq. (12) (labeled 'EA') over a quantum depolarizing channel.

has a 1 at index 0,5 and 8 , then the polynomial is given by 218 $1+x^{5}+x^{8}$. The PCM $H$ has a girth of at least 6 if every 219 difference $\left(d_{i, j_{1}}-d_{i, j_{2}}\right)$ modulo $l$, for $0 \leq i \leq(m-1)$ and 220 $0 \leq j_{1}, j_{2} \leq(\gamma-1)$, is a unique integer between 0 and $(l-1)$. 221 Furthermore, the polynomial transpose is defined as $h_{i}(x)^{T}=222$ $x^{l-d_{i, 1}}+x^{l-d_{i, 2}}+\cdots+x^{l-d_{i, \gamma-1}}$, which would yield the same 223 differences as $h_{i}(x)$. Hence, since in Eq. (9) we are taking the 224 transpose of all the sub-matrices $H_{i}$ and just permuting their 225 location, the differences $\left(d_{i, j_{1}}-d_{i, j_{2}}\right)$ for $H_{z}$ and $H_{x}$ are the 226 same and consequently they both have the same girth ${ }^{3}$.

\section{RESUlts AND Discussions}

To evaluate the performance of our proposed design, we con- 229 sidered the Type-I design of Eq. (2) having $t=15$ and the 230 primitive root $\alpha=2$. Since our design requires $m$ to be even, we 231 arbitrarily chose $m=14$, which yields a [2534, 2172] QLDPC 232 code having a coding rate of 0.857 . We compare our design both 233 to an equivalent bicycle QLDPC code, whose PCM is given by: 234

$$
H_{x}=H_{z}=\left[H_{0}, H_{1}, \ldots, H_{\frac{m}{2}-1}, H_{0}^{T}, H_{1}^{T}, \ldots, H_{\frac{m}{2}-1}^{T}\right],
$$

and to a comparable EA-QLDPC, which requires a single ebit 235 and has:

$$
H_{x}=H_{z}=\left[H_{0}, H_{1}, \ldots, H_{m-1}\right] .
$$

Fig. 1 compares the word error rate (WER) as well as the qubit 237 error rate (QBER) performance of our QLDPC code (labeled 238 'Proposed') to that of the bicycle code of Eq. (11) (labeled 239 ' $\mathrm{BiC}$ ') and to that of the EA-QLDPC code of Eq. (12) (labeled 240 'EA'), when operating over a quantum depolarizing channel. 241 We have evaluated the performance of both the binary as well as 242 the non-binary QLDPC decoder, which operate over the PCMs 243 of Eq. (6) and Eq. (8), respectively. We invoked a maximum 244

\footnotetext{
${ }^{3}$ Following the usual convention [6], we assume that each row of $H_{i}$ is a cyclic right-shift of the row above it. However, if the direction of cyclic shift is reversed, then the resulting PCM $H_{i}$ is symmetric and therefore $H_{i}^{T}=H_{i}$.
} 


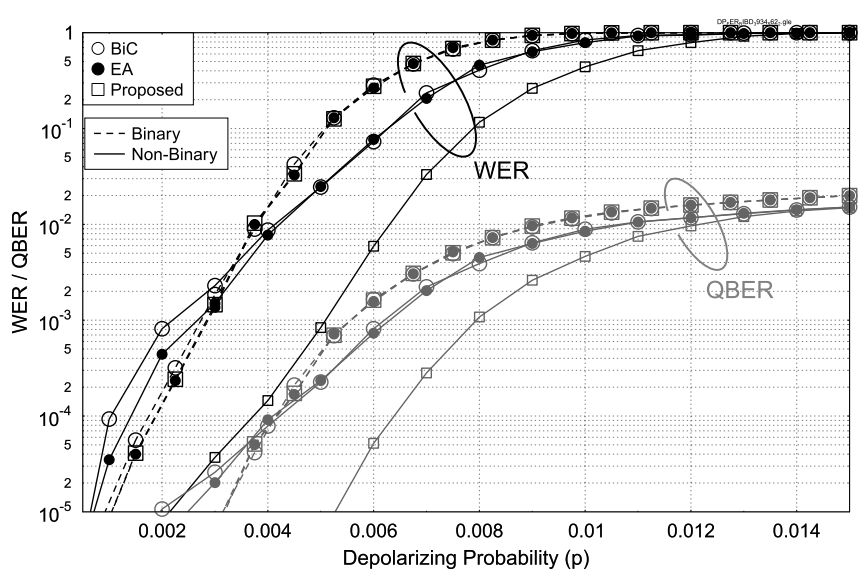

Fig. 2. Comparison of the achievable WER/QBER performance of our proposed [3934, 3372] QLDPC code (labeled 'Proposed') with the bicycle code of Eq. (11) (labeled 'BiC') and the EA-QLDPC code of Eq. (12) (labeled 'EA') over a quantum depolarizing channel. of 100 iterations. Each decoding algorithm iterates until either a valid error is found or the maximum number of iterations is reached. Furthermore, the WER metric here counts the detected as well as the undetected block errors. We may observe in Fig. 1 that the performance of the designed QLDPC is exactly the same as that of the EA-QLDPC code for binary decoding, while that of the bicycle QLDPC code is slightly worse, which is due to the presence of length- 4 cycles. By contrast, when non-binary decoding is invoked, then the performance of our proposed design improves significantly as compared to both the bicycle code as well as the EA-QLDPC. More specifically, the bicycle code achieves a WER of $10^{-4}$ at $p=0.00055$, which increases to $p=0.0007$ when an EA-QLDPC is used. By contrast, our construction exhibits a WER of $10^{-4}$ at $p=0.00215$, which is almost three times better than that of the EA-QLDPC. As discussed in Section III, unlike the bicycle and EA-QLDPC codes, which have numerous short cycles in their GF(4) formalism by virtue of their homogeneous nature, non-homogeneous structures have fewer short cycles in the GF(4) formalism. Consequently, our QLDPC outperforms its comparable bicycle and EA counterparts, when non-binary decoding is invoked.

As an another example, we construct a QLDPC code using the Type-II design of Eq. (2) having $t=14$ and the primitive root $\alpha=3$. The resultant QLDPC code has dimensions of $[3934,3372]$ and a coding rate of 0.857 , when $m=t$. Fig. 2 compares the performance of our non-dual-containing [3934, 3372] QLDPC code both to the comparable bicycle code and to the EA-QLDPC codes. It may be observed that the performance curves of Fig. 2 exhibit the same trend as those of Fig. 1.

\section{CONCLUSIONS}

In this letter, we have conceived a generalized formalism for constructing non-dual-containing CSS-type QLDPC codes from the known classical row-circulant high-rate QC-LDPC codes, which operate efficiently at short and moderate lengths. Since our design is merely based on the transpose and column permutation operations, the characteristics of the underlying classical LDPC matrix are not compromised. In particular, we 282 applied our formalism to the BIBD-based classical LDPCs for 283 evaluating their performance. Furthermore, we invoked both 284 binary as well as non-binary decoding. It was demonstrated 285 that our QLDPC codes have the same performance as the EA- 286 QLDPC codes, when binary decoding is invoked, while they 287 outperform their EA counterparts in case of non-binary decod- 288 ing. As compared to a dual-containing code, our QLDPC codes 289 exhibit a superior performance both for binary as well as for 290 non-binary decoding.

\section{REFERENCES}

[1] P. Botsinis, S. X. Ng, and L. Hanzo, "Quantum search algorithms, 293 quantum wireless, and a low-complexity maximum likelihood iterative 294 quantum multi-user detector design," IEEE Access, vol. 1, pp. 94-122, 295 2013.

[2] A. Beige, B.-G. Englert, C. Kurtsiefer, and H. Weinfurter, "Secure com- 297 munication with single-photon two-qubit states," J. Phys. A Math. Gen., 298 vol. 35, no. 28, p. L407, 2002.

[3] J. Li, X.-B. Chen, G. Xu, Y.-X. Yang, and Z.-P. Li, "Perfect quantum net- 300 work coding independent of classical network solutions," IEEE Commun. 301 Lett., vol. 19, no. 2, pp. 115-118, Feb. 2015.

[4] D. Gottesman, "Stabilizer codes and quantum error correction," Ph.D. 303 dissertation, California Institute of Technology, Pasadena, CA, USA, 304 1997.

[5] H. Tang, J. Xu, Y. Kou, S. Lin, and K. Abdel-Ghaffar, "On algebraic 306 construction of Gallager and circulant low-density parity-check codes," 307 IEEE Trans. Inf. Theory, vol. 50, no. 6, pp. 1269-1279, Jun. 2004.

[6] B. Ammar, B. Honary, Y. Kou, J. Xu, and S. Lin, "Construction of low- 309 density parity-check codes based on balanced incomplete block designs," 310 IEEE Trans. Inf. Theory, vol. 50, no. 6, pp. 1257-1269, Jun. 2004.

[7] S. Johnson and S. R. Weller, "A family of irregular LDPC codes with low 312 encoding complexity," IEEE Commun. Lett., vol. 7, no. 2, pp. 79-81, Feb. 313 2003.

[8] D. MacKay, G. Mitchison, and P. McFadden, "Sparse-graph codes for 315 quantum error correction," IEEE Trans. Inf. Theory, vol. 50, no. 10, 316 pp. 2315-2330, Oct. 2004.

[9] I. B. Djordjevic, "Quantum LDPC codes from balanced incomplete block 318 designs," IEEE Commun. Lett., vol. 12, no. 5, pp. 389-391, May 2008. 319

[10] M.-H. Hsieh, T. A. Brun, and I. Devetak, "Entanglement-assisted quan- 320 tum quasicyclic low-density parity-check codes," Phys. Rev. A, vol. 79, 321 p. 032340, Mar. 2009.

[11] I. B. Djordjevic, "Photonic entanglement-assisted quantum low-density 323 parity-check encoders and decoders," Opt. Lett., vol. 35, no. 9, pp. 1464- 324 1466, May 2010.

[12] R. Bose, "On the construction of balanced incomplete block designs," 326 Ann. Eugenics, vol. 9, pp. 353-399, 1939.

[13] A. R. Calderbank and P. W. Shor, "Good quantum error-correcting codes 328 exist," Phys. Rev. A, vol. 54, no. 2, pp. 1098-1105, Aug. 1996.

[14] A. Steane, "Multiple-particle interference and quantum error correction," 330 Roy. Soc. London Proc. Ser. A, vol. 452, pp. 2551-2577, Nov. 1995.

[15] Y.-J. Wang, B. Sanders, B.-M. Bai, and X.-M. Wang, "Enhanced feedback 332 iterative decoding of sparse quantum codes," IEEE Trans. Inf. Theory, 333 vol. 58, no. 2, pp. 1231-1241, Feb. 2012.

[16] T. Camara, H. Ollivier, and J.-P. Tillich, "A class of quantum LDPC 335 codes: Construction and performances under iterative decoding," in Proc. 336 IEEE Int. Symp. Inf. Theory, Jun. 2007, pp. 811-815.

[17] M. Hagiwara and H. Imai, "Quantum quasi-cyclic LDPC codes," in Proc. 338 Int. Symp. Inf. Theory, Aug. 2010, pp. 806-810. 


\section{QUERY}

Q1: Please check and confirm the insertion of author group and affiliation.

Please provide department name with affiliation as well. 